\title{
Escenarios de aumento del nivel del mar para la costa del Ecuador continental
}

\section{Cenários de aumento do nível do mar na costa do Equador continental}

\author{
Juan Pablo Celemín ${ }^{\mathrm{a}}$ \\ a Investigador adjunto del Consejo Nacional de Investigaciones Científicas y Técnicas (Conicet), \\ doctor en Geografia por la Universidad Nacional del Sur (Argentina). \\ E-mail: jpcelemin@conicet.gov.ar
}

\begin{abstract}
El aumento del nivel del mar plantea escenarios futuros que requieren la atención de la sociedad para que se adopten las medidas de adaptación y mitigación necesarias para hacer frente al problema. La integración de Sistemas de Información Geográfica e imágenes satelitales surge como un elemento fundamental. Para la República del Ecuador la simulación de un incremento de hasta 5 metros del nivel del mar muestra una amplia superficie continental afectada dónde hay importantes centros urbanos. Las provincias de Guayas y Los Ríos aparecen como las principales afectadas, seguidas por El Oro, Esmeraldas y Manabí. Para el caso de Esmeraldas, se realiza un análisis local indicando los tipos de uso de suelo y las parroquias afectadas.

Palabras clave: Ecuador, aumento del nivel del mar, provincia de Esmeraldas, modelos, sistemas de información geográfica.
\end{abstract}

O aumento do nível do mar apresenta cenários futuros que requerem a atenção da sociedade de modo que sejam tomadas as medidas de adaptação e de mitigação necessárias para enfrentar o problema. A integração de Sistemas de Informação Geográfica e imagens de satélite surge como um elemento-chave. No caso da República do Equador, a simulação de um aumento do nível do mar de até 5 metros apresenta uma grande área continental afetada contendo importantes centros urbanos. As províncias de Guayas e Los Rios aparecem como afetadas, seguidas por El Oro, Esmeraldas e Manabí. No caso de Esmeraldas realizou-se uma análise local indicando os tipos de uso da terra e parroquias afetadas.

Palavras-chave: Equador, aumento do nível do mar, província de Esmeraldas, modelos, sistema de informação geográfica. 


\section{INTRODUCCIÓN}

En gran parte del continente americano la costa está amenazada por el aumento en el nivel del mar (ANM), un problema que atrae la atención de investigadores de todo el mundo (FEAGIN; SHERMAN; GRANT, 2005). Para poder evaluar estos riesgos en forma efectiva, las ciudades costeras están usando modelos para simular los escenarios propuestos por el Panel Intergubernamental del Cambio Climático (Intergovernmental Panel on Climate Change - IPCG). Se estima que 1,200 millones de personas o aproximadamente el $23 \%$ de la población mundial, vive a menos de $100 \mathrm{~m}$ de altura sobre el nivel del mar y a $100 \mathrm{~km}$. de una costa y, por lo tanto, son vulnerables al aumento en el nivel del mar (COOPER; BEEVERS; OPPENHEIMER, 2005). Un análisis global de la población y de los asentamientos urbanos en Zonas Costeras de Baja Elevación, es decir, el área contigua a la costa que se encuentra a menos de $10 \mathrm{~m}$ sobre el nivel del mar, encontró que cubre el $2 \%$ del terreno del mundo, pero contiene el $10 \%$ de la población mundial y el $13 \%$ de la población urbana (MCGRANAHAN et al., 2007).

El IPCC sugirió que, para el año 2100, se produciría un aumento de 0,09 a 0,88 m en el nivel del mar, excepto que se reduzcan sustancialmente las emisiones de gases de invernadero (IPCC, 2001). Estudios más recientes indican que el derretimiento de las capas de hielo podría ser más rápido de lo previsto y, por lo tanto, presentar un mayor desafío para la sociedad (OVERPEGK et al., 2006). Los datos actuales de descarga de hielo demuestran que el derretimiento del hielo puede provocar un aumento en el nivel del mar de 1 a 3 m para el año 2100. Nuevos datos sobre las tasas de desglaciación, en Groenlandia y Antártida, sugieren un mayor impacto del derretimiento glacial, $\mathrm{y}$, posiblemente, se tengan que revisar las estimaciones superiores de ANM para este siglo. Un documento de trabajo sobre del Banco Mundial (DASGUPTA et al., 2007) cita numerosos autores, como Rignot y Kanagaratnam (2006), Hanna et al. (2008) y Krabill et al. (2004) que concluyen que la contribución de la capa de hielo de Groenlandia al ANM es, aproximadamente, el doble de la tasa que supone el informe del IPCG. Otros estudios indican que un colapso en la capa de hielo de Antártida Occidental elevaría el nivel del mar en promedio unos 5 a $6 \mathrm{~m}$ (TOL et al., 2006, apud DASGUPTA et al., 2007). En forma similar, Velicogna y Wahr (2006) señalan que la capa de hielo en la Antártida se está reduciendo más rápido de lo que sugiere el IPGC.

Dasgupta et al. (2007) aporta una perspectiva más amplia sobre el problema del ANM al modelar nuevos datos. Sus investigaciones sobre planificación precautoria sugieren una gama realista de 1 a $3 \mathrm{~m}$ de ANM. Se calcularon los modelos usando Sistemas de Información Geográfica (SIG) e imágenes de satélite (SRTM $90 \mathrm{~m}$ ) para escenarios de ANM que oscilan entre 1 y $5 \mathrm{~m}$.

La distribución espacial del impacto del cambio climático no será uniforme. Se espera que algunos cambios climáticos regionales sean mucho más pronunciados que los cambios en el promedio global, y otros menos (CBO, 2005). Además del ANM, se sabe que los riesgos inducidos por el cambio climático afectarán la salud humana de varias maneras y por lo menos se duplicarán para el año 2030 (PATZ et al. 2005).

Si bien no existen modelos capaces de capturar la gama completa del impacto del ANM, pueden proporcionar los primeros pasos básicos para mitigar dichos impactos. Por ejemplo, el riesgo de inundación se puede mitigar a bajo costo con planificación urbana y regional. Las iniciativas usan nuevos mapas de riesgo de inundaciones y modificaciones al código de edificación para tener en cuenta el cambio climático. El costo futuro de reducir el impacto del cambio climático también se puede moderar tomando medidas para reducir las emisiones de gases de invernadero, lo que debe seguir como parte principal del enfoque global. La reducción de gases de invernadero, sin embargo, simplemente retardará los efectos del cambio climático en el próximo siglo, pero no los prevendrá ni los revertirá. Sin embargo, ello permitirá ganar tiempo para implementar las adaptaciones necesarias para proteger a la sociedad de los efectos del cambio climático (HERWEIJER et al. 2008).

Las ciudades que realizan esfuerzos de gestión de la costa deberían tener en cuenta estrategias no estructurales, tales como la sustentación de playas, la construcción y la estabilización de dunas, así como iniciativas estructurales (HERWEIJER et al. 2008). Sin embargo, todavía hay una gran cantidad de información confusa. $\mathrm{Al}$ hacer distintas interpretaciones de la 
misma base de conocimientos, hay "optimistas" que opinan que la adaptación humana reducirá la magnitud del impacto a un nivel tal que el ANM se convertirá en un problema trivial. Este argumento parece una versión modificada de la idea de que la contaminación no es un problema importante porque en el futuro la humanidad tendrá los recursos para eliminarla. Por el otro lado, los "pesimistas" opinan que el ANM y el cambio climático son una amenaza crítica en el siglo XXI (NICHOLLS; TOL, 2006).

Al tratar de atacar el problema del cambio climático, los investigadores y los dirigentes políticos tienen que lidiar con varias fuentes de incertidumbre. Todavía hay barreras que superar antes de poder realizar un análisis integral de impacto y respuesta: el conocimiento incompleto de los procesos relevantes afectados por el ANM y sus interacciones, los datos insuficientes sobre las condiciones existentes, la dificultad para elaborar escenarios locales y regionales de cambios futuros, y la falta de metodologías analíticas apropiadas para evaluar algunos de los impactos (NICHOLLS; MIMURA, 1998). Si bien la consecuencia más traumática del ANM es la inundación permanente, hay otros procesos visibles que se deben considerar, como la erosión de la costa, las inundaciones episódicas debido a oleadas de tormentas y la salinización de acuíferos, los cuales, en algunos casos, requieren una atención más urgente.

La Comisión Económica para América Latina y el Caribe publicó un trabajo muy detallado en el que plantea el impacto del ANM para la región (CEPAL, 2015). En dicho trabajo se sostiene que los análisis realizados para la región sobre el comportamiento histórico de las dinámicas costeras identifican una inequívoca tendencia del aumento del nivel del mar en todos los puntos de América Latina y el Caribe. Destaca que, además del ANM, se debe considerar que su combinación con la alteración de las dinámicas de otras variables - además de los procesos relacionados con fenómenos extremos (los huracanes y el fenómeno de El Niño/Oscilación Austral) -, resultará en el aumento de la complejidad de los impactos y la vulnerabilidad de los sistemas socioeconómicos y ecológicos de cualquier país de la región. Asimismo, las características físicas y socioeconómicas de la región imponen retos adicionales que pueden incrementar los efectos asociados al ANM al ser mayoritariamente urbana (el $80 \%$ de su población se concentra en ciudades). Además, en las tendencias registradas y en todos los escenarios socioeconómicos establecidos por el IPGG se prevé, en mayor o menor medida, un incremento considerable de la población en las zonas costeras. Esto lleva aparejada la construcción de nuevas infraestructuras, la introducción de industrias extractivas, la reducción de los recursos de agua dulce, el mayor aporte de sedimentos y otras problemáticas asociadas a la conservación de hábitats costeros (CEPAL, 2015, p. 41).

Con base en lo que se expresó anteriormente, el presente trabajo modeliza los distintos escenarios de ANM para la costa continental de la República del Ecuador, con énfasis en la provincia de Esmeraldas, al norte del país. Para todo el país, el estudio se centra en la escala provincial mientras que para Esmeraldas se utiliza una escala más detallada que es constituida por las parroquias. Asimismo, se analizan los tipos de uso de suelo afectados de acuerdo a los criterios del IPCC (Uso de Suelo Nivel 2). Consiguientemente, se obtiene un análisis a escala local, con un buen nivel de detalles y acompañado por cartografía que facilita la interpretación de los diferentes escenarios de ANM para el área de estudio en un rango de incremento entre 0 y $5 \mathrm{~m}$.

\section{ÁreA DE ESTUdio}

La Republica del Ecuador es uno de los países más pequeños de América Latina con una superficie de $283.591 \mathrm{~km}^{2}$ y una línea de costa de $2.237 \mathrm{~km}$. Su población total es de 14.483.499 (INEC, 2015) habitantes. Limita al norte con Colombia y al sur y al este con Perú (Figura 1).

Por su parte, la provincia de Esmeraldas posee una superficie de 16.132,23 km² y una población de 534.092 habitantes. Está compuesta por siete cantones y 64 parroquias (Figura 2 y Cuadro 1). 
Figura 1. Localización y provincias del Ecuador continental ${ }^{1}$.

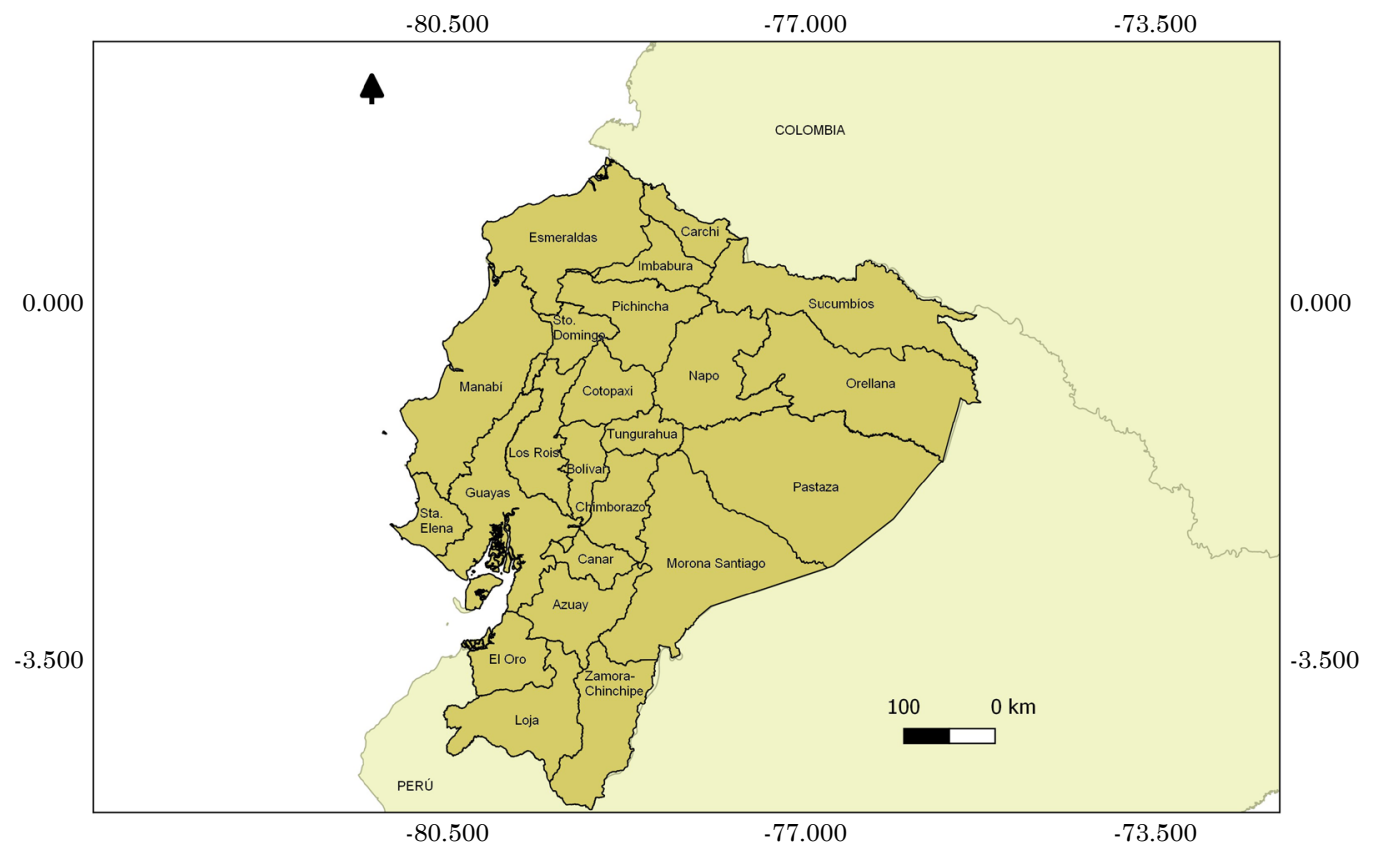

Fuente: Adaptado de la cartografía del Ministerio del Ambiente de Ecuador (MAE).

Figura 2. Localización de las parroquias de la Provincia de Esmeraldas. $-80.000$ $-79.000$

$-78.000$

$1.000-$

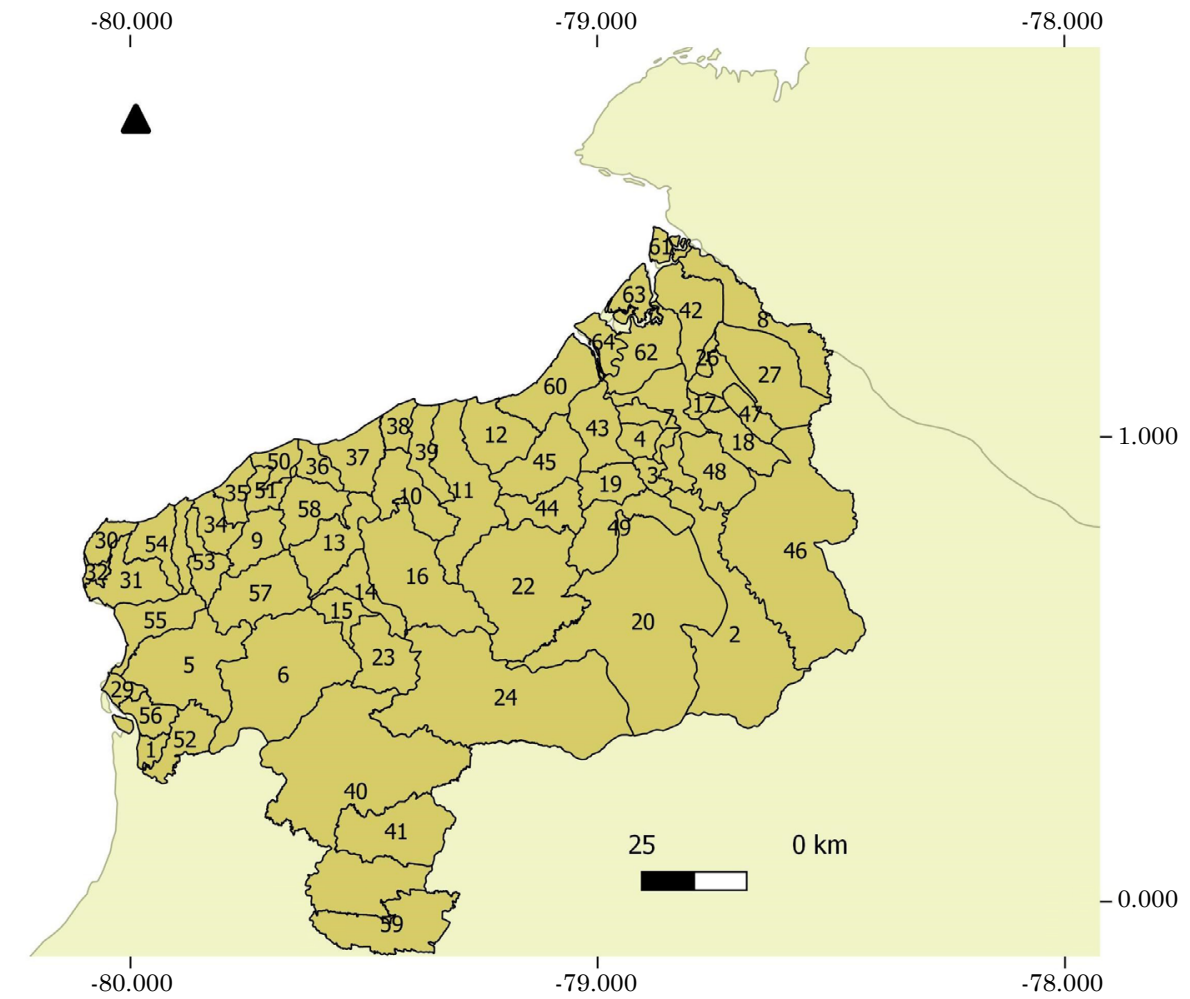

Fuente: Adaptado de la cartografía del MAE.

1 En el 2007 se crea la provincia de Santa Elena en el extremo occidental de la provincia de Guayas. Para este trabajo permanecen integradas debido a la cartografía original, provista por el Ministerio del Medio Ambiente del Ecuador. 
Cuadro 1. Parroquias de la Provincia de Esmeraldas, Ecuador.

\begin{tabular}{|c|c|c|c|c|c|c|c|}
\hline \begin{tabular}{|c|} 
Pquia. \\
Nro. \\
\end{tabular} & NOMBRE & $\begin{array}{l}\text { Pquia. } \\
\text { Nro. }\end{array}$ & NOMBRE & $\begin{array}{l}\text { Pquia. } \\
\text { Nro. }\end{array}$ & NOMBRE & $\begin{array}{l}\text { Pquia. } \\
\text { Nro. }\end{array}$ & NOMBRE \\
\hline 1 & SALIMA & 17 & $\begin{array}{l}\text { SAN JAVIER DE } \\
\text { CACHAVI }\end{array}$ & 33 & $\begin{array}{c}\text { SUA (CAB EN LA } \\
\text { BOCANA) }\end{array}$ & 49 & $\begin{array}{l}\text { ATAHUALPA } \\
\text { (CAB. EN } \\
\text { CAMARONES) }\end{array}$ \\
\hline 2 & $\begin{array}{l}\text { LUIS V. TORRES } \\
\text { (CAB. EN PLAYA } \\
\text { DE ORO) }\end{array}$ & 18 & URBINA & 34 & ATACAMES & 50 & ESMERALDAS \\
\hline 3 & SELVA ALEGRE & 19 & $\begin{array}{c}\text { SAN JOSE DE } \\
\text { CAYAPAS }\end{array}$ & 35 & TONSUPA & 51 & VUELTA LARGA \\
\hline 4 & $\begin{array}{c}\text { COLON ELOY DEL } \\
\text { MARIA }\end{array}$ & 20 & TELEMBI & 36 & TACHINA & 52 & $\begin{array}{l}\text { SAN JOSE DE } \\
\text { CHAMANGA }\end{array}$ \\
\hline 5 & SAN GREGORIO & 21 & CARONDELET & 37 & $\begin{array}{l}\text { CAMARONES } \\
\text { (CAB. EN SAN } \\
\text { VICENTE) }\end{array}$ & 53 & LA UNION \\
\hline 6 & CUBE & 22 & $\begin{array}{l}\text { SANTO DOMINGO } \\
\text { DE ONZOLE }\end{array}$ & 38 & RIOVERDE & 54 & TONCHIGUE \\
\hline 7 & CONCEPCION & 23 & $\begin{array}{c}\text { CHURA } \\
\text { (CHANCAMA) } \\
\text { (CAB. EN EL } \\
\text { YERBERO) }\end{array}$ & 39 & ROCAFUERTE & 55 & MUISNE \\
\hline 8 & $\begin{array}{l}\text { MATAJE (CAB EN } \\
\text { SANTANDER) }\end{array}$ & 24 & MALIMPIA & 40 & $\begin{array}{l}\text { ROSA ZARATE } \\
\text { (QUININDE) }\end{array}$ & 56 & DAULE \\
\hline 9 & TABIAZO & 25 & MALDONADO & 41 & LA UNION & 57 & $\begin{array}{l}\text { CRNEL. CARLOS } \\
\text { C. TORRES (CAB. } \\
\text { EN HUELE) }\end{array}$ \\
\hline 10 & CHONTADURO & 26 & CALDERON & 42 & SAN LORENZO & 58 & SAN MATEO \\
\hline 11 & $\begin{array}{l}\text { MONTALVO (CAB } \\
\text { EN HORQUETA) }\end{array}$ & 27 & $\begin{array}{l}\text { TULULBI (CAB } \\
\text { EN RICAURTE) }\end{array}$ & 43 & BORBON & 59 & LA CONCORDIA \\
\hline 12 & LAGARTO & 28 & TIMBIRE & 44 & $\begin{array}{c}\text { SAN FRANCISCO } \\
\text { DE } \\
\text { ONZOLE }\end{array}$ & 60 & LA TOLA \\
\hline 13 & CHINCA & 29 & BOLIVAR & 45 & ANCHAYACU & 61 & $\begin{array}{c}\text { ANCON } \\
\text { (PICHANGAL) } \\
\text { (CAB. EN PALMA } \\
\text { REAL) }\end{array}$ \\
\hline 14 & MAJUA & 30 & GALERA & 46 & $\begin{array}{l}\text { ALTO TAMBO } \\
\text { (CAB EN } \\
\text { GUADUAL) }\end{array}$ & 62 & TAMBILLO \\
\hline 15 & VICHE & 31 & SAN FRANCISCO & 47 & SANTA RITA & 63 & $\begin{array}{l}\text { PAMPANAL DE } \\
\text { BOLIVAR }\end{array}$ \\
\hline 16 & CHUMUNDE & 32 & $\begin{array}{c}\text { QUINGUE } \\
\text { (OLMEDO } \\
\text { PERDOMO } \\
\text { FRANCO) } \\
\end{array}$ & 48 & $\begin{array}{c}5 \text { DE JUNIO } \\
\text { (CAB EN UIMBI) }\end{array}$ & 64 & $\begin{array}{l}\text { VALDEZ } \\
\text { (LIMONES) }\end{array}$ \\
\hline
\end{tabular}

Fuente: MAE.

\section{MetodoloGía}

La principal complejidad de este tipo de trabajo radica en la necesidad de integrar los formatos raster y vectorial. Mientras que las imágenes satelitales se encuentran en formato raster, es necesario convertirlas al tipo vectorial para poder realizar las operaciones de geoprocesamiento (cortes e intersección de vectores) para poder obtener la cartografía y los datos cuantitativos, que muestren los distintos escenarios planteados. 


\section{Elaboración del modelo digital de terreno}

El instrumento principal para modelar el ANM es el modelo de elevación digital (Digital Evaluation Model, o DEM). Para ello, se utilizó la información provista por la Misión Topográfica de Radar del Transbordador
(Shuttle Radar Topography Mission - SRTM, con una resolución de $30 \mathrm{~m})^{2}$, que permitió obtener un DEM para todo el Ecuador (Figura 3). La imagen fue extraída del sitio Earth Explorer, que permite acceder a información satelital de manera gratuita.

Figura 3. DEM para la República del Ecuador (en metros).

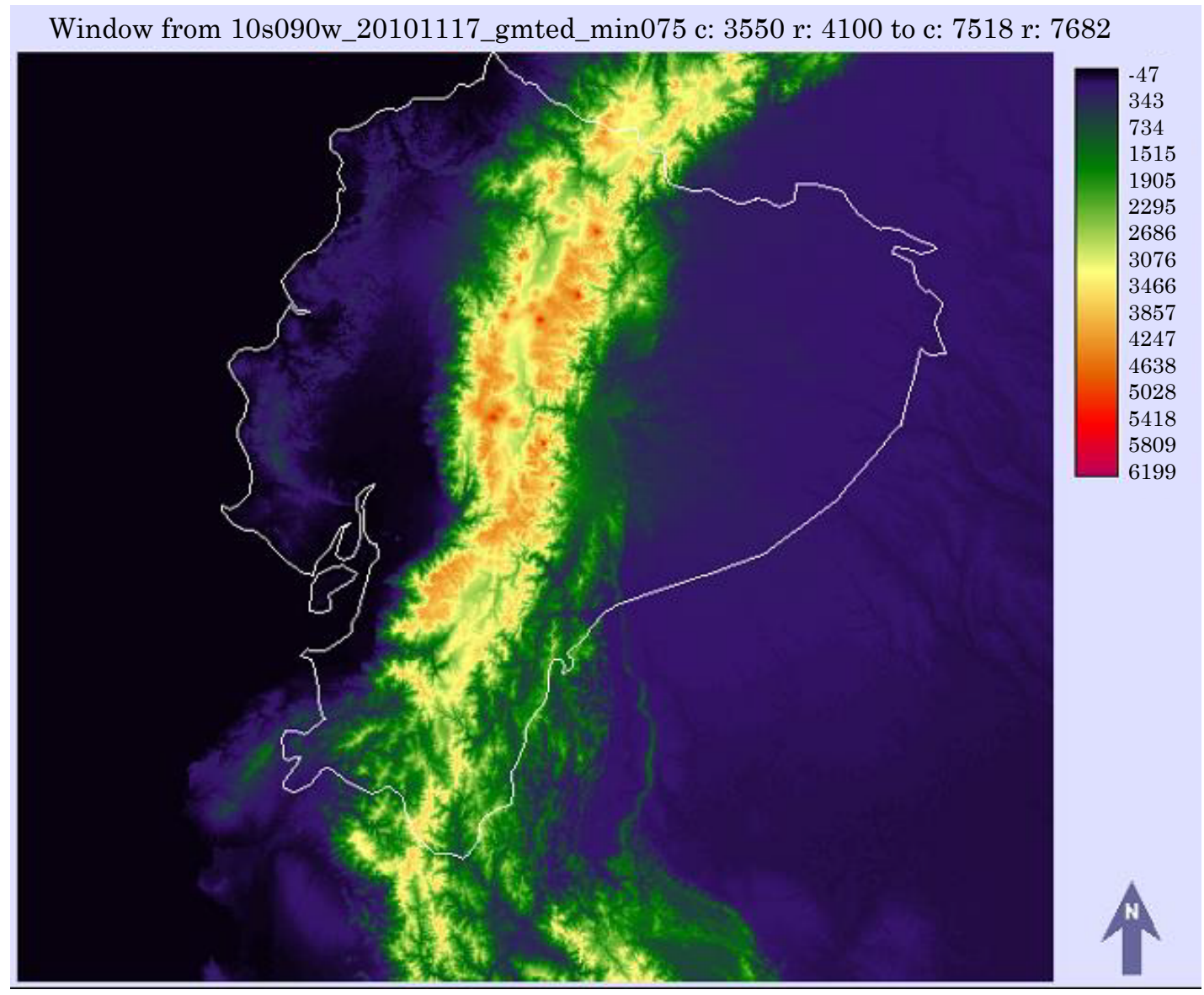

Fuente: Adaptado de imagen SRTM (diciembre 2016).

Con posterioridad, la imagen del DEM fue convertida a formato vectorial para poder procesar los escenarios de ANM $(0$ a $1 ; 1$ a $2 ; 2$ a $3 ; 3$ a 4 y 4 a $5 \mathrm{~m})$. Se creó un archivo vectorial para cada uno de los escenarios de ANM, que fueron mencionados con anterioridad, para luego cruzarlos con las provincias del país y con las parroquias de Esmeraldas. De esta manera, se obtiene la superficie potencial de ANM, para cada escala de análisis, de acuerdo a los distintos rangos de ANM.

El Ministerio del Medio Ambiente del Ecuador (MAE) proveyó la cartografía en formato vectorial, que incluye los distintos tipos de suelo categorizados por el IPCG para la provincia de Esmeraldas, que permite realizar un estudio más detallado a escala local para dicha jurisdicción.

\section{Resultados}

\section{ANM para la costa de la República del Ecuador continental}

La Figura 4 permite una interpretación visual de cómo el ANM afecta a cada una de las provincias:

2 Este satélite, también, posee imagines con resolución espacial de $90 \mathrm{~m}$. 
Figura 4. Escenarios de ANM en metros para las provincias de la costa del Ecuador continental.

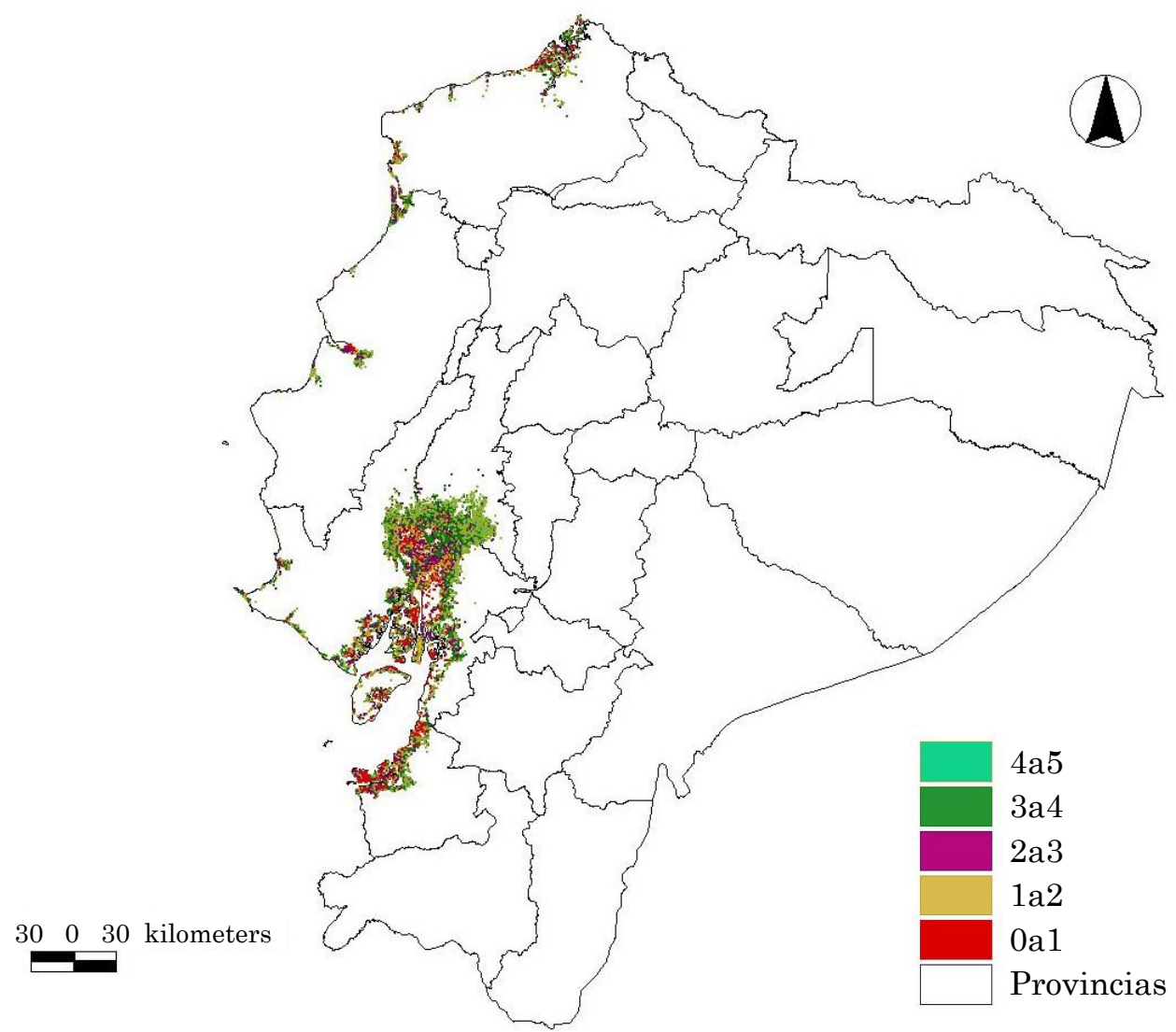

Fuente: Adaptado del DEM y de la cartografía del MAE.

Se puede observar como la parte sur del Ecuador se vería más afectada que la norte, ante casos de ANM. No obstante, es necesario hacer un análisis más detallado para cada provincia, de acuerdo a los distintos escenarios. La Tabla 1 registra, para cada provincia, la superficie en $\mathrm{km}^{2} \mathrm{y}$ su respectivo porcentaje que estaría afectado por el ANM, para cada uno de los rangos considerados. Por su parte, la Tabla 2 muestra el acumulado de 0 a $5 \mathrm{~m}$.
Los distintos escenarios muestran que las provincias más afectadas ante los distintos escenarios son GuayasSanta Elena, Los Ríos y el Oro (en ese orden). Se destacan las dos primeras con más del 10\% de su superficie afectada. En el caso de Guayas, la ciudad de Guayaquil, la más populosa del país, se vería afectada directamente. Por lo tanto, es una de las localidades en el continente que más podría padecer los efectos del ANM.

Tabla 1. Superficie en $\mathrm{km}^{2}$ por provincia afectada por incremento del nivel del mar de 0 a $1 \mathrm{~m}$.

\begin{tabular}{lccccccccccc}
\hline Provincia & Superficie & Sup0a1 & \% & Sup1a2 & \% & Sup2a3 & \% & Sup3a4 & \% & Sup4a5 & \% \\
\hline El Oro & $5.674,11$ & 206,40 & 3,64 & 67,21 & 1,18 & 57,33 & 1,01 & 54,73 & 0,96 & 62,51 & 1,10 \\
Esmeraldas & $152.115,25$ & 78,09 & 0,05 & 94,20 & 0,06 & 80,88 & 0,05 & 74,08 & 0,05 & 65,62 & 0,04 \\
Guayas y & $19.181,91$ & 662,55 & 3,45 & 642,19 & 3,35 & 526,41 & 2,74 & 412,07 & 2,15 & 346,69 & 1,81 \\
Santa Elena & & & & & & & & & & & \\
Los Ríos & $7.207,73$ & 73,60 & 1,02 & 112,26 & 1,56 & 157,70 & 2,19 & 200,92 & 2,79 & 210,20 & 2,92 \\
Manabí & $18.609,78$ & 39,94 & 0,21 & 61,32 & 0,33 & 51,10 & 0,27 & 40,84 & 0,22 & 36,23 & 0,19 \\
\hline
\end{tabular}

Fuente: DEM. 
Tabla 2. Superficie en $\mathrm{km}^{2}$ por provincia afectada por incremento del nivel del mar de 0 a $5 \mathrm{~m}$.

\begin{tabular}{lcccc}
\hline Provincia & Población & Superficie & Sup0a5 & \% \\
\hline El Oro & 778.115 & $5.674,11$ & 448,18 & 7,90 \\
Esmeraldas & 534.092 & $152.115,25$ & 392,87 & 0,26 \\
Guayas y & 3.954 .176 & $19.181,91$ & $2.589,91$ & 13,50 \\
Santa Elena & & & & \\
Los Ríos & 865.340 & $7.207,73$ & 754,68 & 10,47 \\
Manabí & 1.369 .780 & $18.609,78$ & 229,43 & 1,23 \\
\hline
\end{tabular}

Fuente: DEM y Instituto Nacional de Estadísticas y Censos (2015).

\section{Análisis específico para las parroquias de la Provincia de Esmeraldas}

En la Figura 5 se observa que, para la provincia de Esmeraldas, para un ANM extremo de 0 a $5 \mathrm{~m}$, la mayor parte de la superficie afectada se concentra en el norte y, en menor medida, en el sur. La Tabla 3 permite reconocer aquellas parroquias más afectadas para los distintos rangos de ANM considerados.
El ANM afectara principalmente a las Parroquias de Ancón, San Lorenzo, Pampanal de Bolívar, Valdez, La Tola con más de un tercio de su superficie comprometida para el escenario más extremo de $5 \mathrm{~m}$. El cruce con la cartografía provista por el Ministerio del Ambiente del Ecuador facilita, además, reconocer los tipos de suelo que se verían afectados. De acuerdo con el IPCC, los usos de suelo del Nivel 2 se clasifican en las siguientes categorías:

Área poblada, Infraestructura, Cuerpo de agua artificial, cuerpo de agua natural, Bosque nativo, Plantación forestal, Cultivo anual, Cultivo permanente, Cultivo semipermanente, Mosaico agropecuario, Pastizal, Vegetación arbustiva, Vegetación herbácea.

Dichas categorías fueron cruzadas con la superficie de las parroquias de la provincia de Esmeraldas, comprendida entre 0 y $5 \mathrm{~m}$, que puede ser afectada por el ANM (figura 6).

Un análisis más detallado se encuentra en la Tabla 4, que permite reconocer la superficie afectada de cada uso de suelo en $\mathrm{km}^{2}$.

Figura 5. Superficie de 0 a $5 \mathrm{~m}$ afectada por incremento del nivel del mar para las parroquias costeras de la provincia de Esmeraldas.

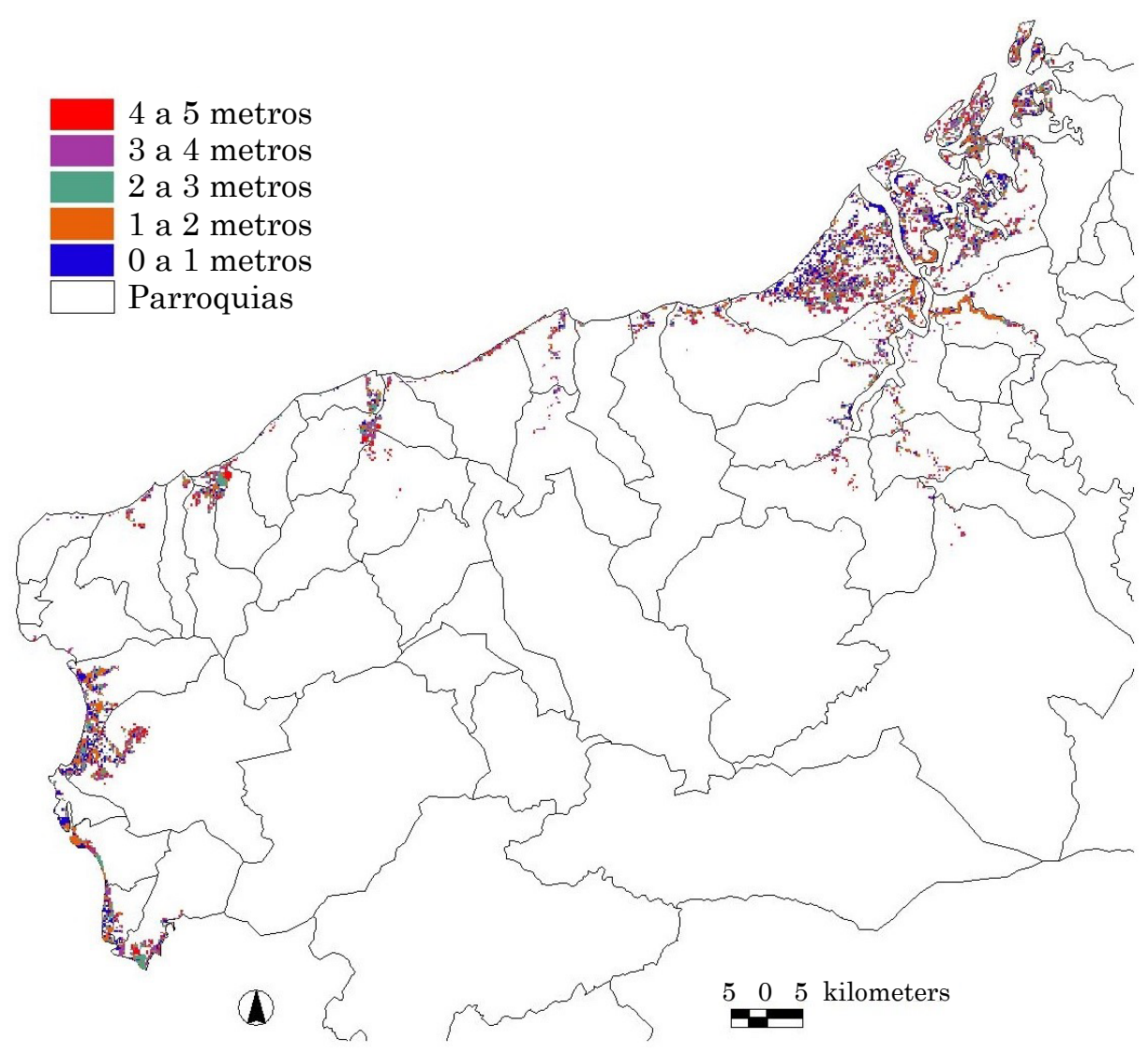

Fuente: Adaptado del DEM y de la cartografía del MAE. 
Tabla 3. Porcentaje de superficie de parroquia afectada por incremento del nivel del mar para la Provincia de Esmeraldas para distintos escenarios.

\begin{tabular}{|c|c|c|c|c|c|c|}
\hline Parroquias Afectadas & $\% 0 a 1$ & $\% 1 \mathrm{a} 2$ & $\% 2 a 3$ & $\% 3 a 4$ & $\% 4 a 5$ & $\% 0 \mathrm{a5}$ \\
\hline Ancón (Pinchangal) Cab. En Palma Real) & 8,1 & 19,1 & 15,1 & 8,2 & 6,7 & 57,2 \\
\hline San Lorenzo & 20,2 & 22,9 & 22,5 & 16,8 & 7,3 & 89,7 \\
\hline Mataje (Cab. En Santander) & 0,4 & 0,7 & 1,4 & 0,7 & 0,6 & 3,8 \\
\hline Pampanal De Bolívar & 4,7 & 10,2 & 11,4 & 11,0 & 8,7 & 46,0 \\
\hline Mataje (Cab. En Santander) & 0,4 & 0,7 & 1,4 & 0,7 & 0,6 & 3,8 \\
\hline Tambillo & 4,6 & 4,7 & 5,5 & 4,9 & 4,0 & 23,7 \\
\hline Valdez (Limones) & 13,2 & 10,5 & 9,2 & 9,8 & 8,5 & 51,2 \\
\hline La Tola & 10,1 & 8,4 & 6,5 & 5,5 & 4,6 & 35,1 \\
\hline Concepción & 0,2 & 0,6 & 0,2 & 0,4 & 0,4 & 1,8 \\
\hline Borbón & 3,4 & 7,0 & 3,4 & 5,1 & 3,3 & 22,2 \\
\hline Maldonado & 0,4 & 8,0 & 1,4 & 1,8 & 1,6 & 13,2 \\
\hline Lagarto & 0,3 & 0,5 & 0,5 & 0,6 & 0,9 & 2,8 \\
\hline Montalvo (Cab. En Horqueta) & 0,2 & 0,2 & 0,2 & 0,2 & 0,2 & 1,0 \\
\hline Rioverde & 0,9 & 1,4 & 1,4 & 2,2 & 2,1 & 8,0 \\
\hline Rocafuerte & 0,0 & 0,0 & 0,2 & 0,2 & 0,2 & 0,6 \\
\hline Camarones (Cab. En San Vicente) & 0,1 & 0,3 & 0,3 & 0,5 & 0,6 & 1,8 \\
\hline Anchayacu & 0,6 & 0,6 & 1,0 & 0,8 & 0,5 & 3,5 \\
\hline San Jose De Cayapas & 0,4 & 0,8 & 0,7 & 0,9 & 1,2 & 4,0 \\
\hline Esmeraldas & 1,8 & 3,2 & 3,5 & 2,2 & 1,3 & 12,0 \\
\hline Tachina & 0,3 & 0,1 & 0,4 & 1,2 & 1,2 & 3,2 \\
\hline Chontaduro & 0,1 & 0,1 & 0,1 & 0,3 & 0,2 & 0,8 \\
\hline Tonsupa & 0,2 & 0,0 & 0,1 & 0,1 & 0,6 & 1,0 \\
\hline Vuelta Larga & 0,4 & 0,7 & 1,2 & 1,6 & 1,0 & 4,9 \\
\hline San Jose De Cayapas & 0,4 & 0,8 & 0,7 & 0,9 & 1,2 & 4,0 \\
\hline San Francisco De Onzole & 0,0 & 0,0 & 0,1 & 0,4 & 0,6 & 1,1 \\
\hline Atahualpa (Cab.En Camarones) & 0,1 & 0,1 & 0,0 & 0,1 & 0,1 & 0,4 \\
\hline Atacames & 1,0 & 2,1 & 3,6 & 1,6 & 2,2 & 10,5 \\
\hline Tonchigue & 0,1 & 0,3 & 0,3 & 0,6 & 0,8 & 2,1 \\
\hline La Unión & 0,3 & 1,1 & 0,8 & 0,9 & 0,9 & 4,0 \\
\hline Muisne & 5,3 & 6,2 & 3,4 & 1,9 & 1,1 & 17,9 \\
\hline San Gregorio & 0,9 & 1,2 & 0,8 & 0,8 & 0,9 & 4,6 \\
\hline Bolívar & 5,5 & 3,0 & 1,1 & 0,9 & 0,5 & 11,0 \\
\hline Daule & 0,6 & 3,3 & 1,8 & 1,0 & 0,9 & 7,6 \\
\hline San Jose De Chamanga (Cab. En Chamanga) & 0,9 & 1,0 & 2,6 & 1,6 & 1,7 & 7,8 \\
\hline
\end{tabular}

Fuente: DEM y cartografía del MAE. 
Figura 6. Tipo de uso de suelo en la provincia de Esmeraldas en el rango de 0 a $5 \mathrm{~m}$.

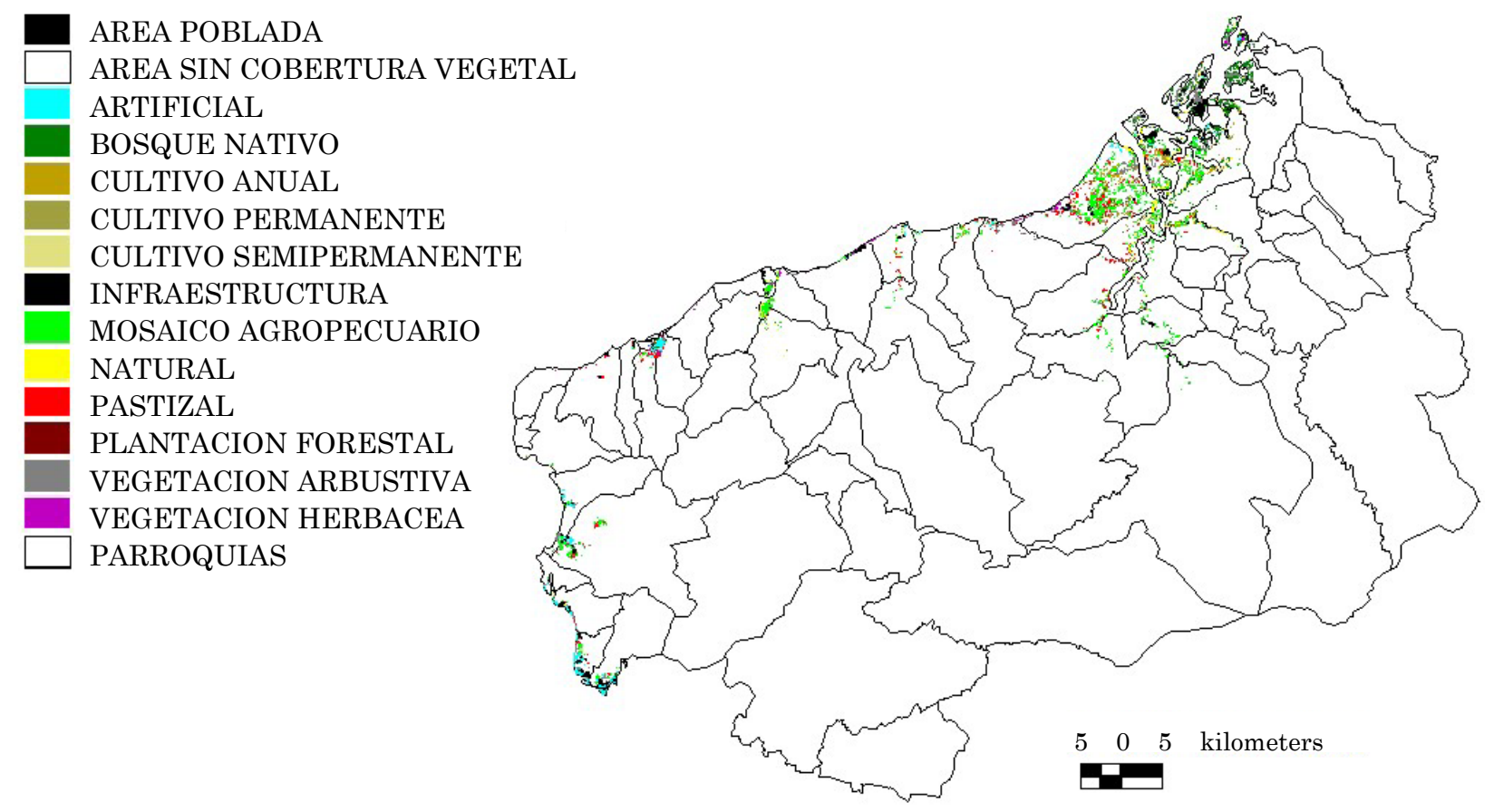

Fuente: Adaptado del DEM y de la cartografía del MAE.

Tabla 4 - Superficie de Uso de Suelo de la provincia de Esmeraldas que fue afectada por aumento del nivel del mar de 0 a $5 \mathrm{~m}$, según criterios del IPCC, Nivel 2.

\begin{tabular}{lc}
\hline Uso IPCC Nivel 2 & km2 \\
\hline Área Poblada & 7.302 .432 \\
Área sin Cobertura Vegetal & 16.532 .303 \\
Cuerpo de Agua Artificial & 23.389 .594 \\
Bosque Nativo & 47.386 .191 \\
Cultivo Anual & 17.518 .721 \\
Cultivo Permanente & 9.164 .931 \\
Cultivo Semipermanente & 1.755 .434 \\
Infraestructura & 0.108 .333 \\
Mosaico Agropecuario & 91.812 .583 \\
Cuerpo de Agua Natural & 1.955 .043 \\
Pastizal & 38.553 .222 \\
Plantación forestal & 147.265 \\
Vegetación Arbustiva & 49.062 .497 \\
Vegetación Herbácea & 7.550 .741 \\
Total & 329.834 .677 \\
\hline
\end{tabular}

Fuente: DEM y cartografía del MAE.

Para el Uso de Suelo Nivel 2 se observa que el Área Poblada afectada por el ANM, supera los $7 \mathrm{~km}^{2}$, a la vez que el Mosaico Agropecuario impactado superaría los $91 \mathrm{~km}^{2}$. Es decir, un ANM de este rango afectaría las condiciones de vida de las personas al impactar en sus centros urbanos y en la producción primaria.

\section{Consideraciones FInALES}

Existen numerosas incertidumbres que están asociadas al impacto del cambio climático a escala global y regional. No obstante, es ineludible que el nivel del mar aumentará en las próximas décadas. Queda por conocer aún la magnitud de dicho fenómeno. Es por eso que surge la necesidad de plantear diferentes escenarios, tanto conservadores como más extremos, que representen la magnitud del potencial problema.

Este tipo de trabajos, con un enfoque regional y local, permite conocer con más detalle los distintos escenarios y, a partir de la elaboración de cartografía y tablas, distinguir aquellas zonas más afectadas. Se observa que, para la República del Ecuador en su litoral costero continental, la provincia de Guayas (que contiene a la populosa localidad de Guayaquil) sería la más afectada, seguida por los Ríos y El Oro.

Un estudio más detallado a escala local, a nivel de parroquias, muestra para la provincia de Esmeraldas al norte 
del país un total del 2,58\% de su superficie afectada por el ANM para su escenario más radical (hasta $5 \mathrm{~m}$ ). Algunas parroquias verían gran parte de su superficie afectada por el ANM, tal es el caso de Ancón, San Lorenzo o Valdez, entre otras. El cruce con cartografía existente relacionada con usos de suelo permite reconocer más de $7 \mathrm{~km}^{2}$ de Área Poblada afectada y más de $158 \mathrm{~km}^{2}$ de Mosaico Agropecuario, impactando directamente sobre las condiciones de vida y actividades económicas de las personas de la zona.

Dado que los escenarios son de largo plazo, se está a tiempo de adoptar las medidas estructurales y no estructurales necesarias para una gestión costera que permita mitigar los efectos del ANM. Para ello, se requiere de un equipo interdisciplinario y más trabajos de investigación que simulen escenarios a distintas escalas (urbanas y regionales). Un diagnóstico adecuado del problema, apoyado en el uso de geotecnologías, constituye el paso inicial para encararlo de manera apropiado y, así, plantear estrategias y medidas para paliar sus efectos sobre el ambiente, la sociedad y la economía local en la medida de lo posible.

\section{Agradecimientos}

El presente trabajo fue patrocinado por el Proyecto Prometeo de la Secretaría de Educación Superior, Ciencia, Tecnología e Innovación de la República del Ecuador, con lugar de trabajo en el Ministerio del Medio Ambiente del país, durante el año 2014.

\section{REFERENCIAS}

CBO - CONGRESSIONAL BUDGET OFFICE. Uncertainty in analyzing climate change: policy implications. Washington, DG: Congressional Budget Office, 2005.

GEPAL - COMISIÓN ECONÓMICA PARA AMÉRICA LATINA. La economía del cambio climático en América Latina y el Garibe. Santiago de Chile: CEPAL, 2015.

GOOPER, M. J. P.; BEEVERS, M. D.; OPPENHEIMER, M. Future sea level rise and the New Jersey coast: assessing potential impacts and opportunities. Princeton: Woodrow Wilson School of Public and International Affairs, 2005.
DASGUPTA, S. et al. The impact of sea level rise on developing countries: a comparative analysis. Washington, DC: World Bank Policy, 2007.

FEAGIN, R. A.; SHERMAN, D. J.; GRANT, W. E. Coastal erosion, global sea-level rise, and the loss of sand dune plant habitats. Frontiers in Ecology and the Environment, Washington, DG, v. 3, n. 7 , p. 359-364, 2005.

HANNA, E. et al. Increased runoff from melt from the Greenland ice sheet: a response to global warming. Journal of Climate, Boston, v. 21, n. 2, p. 331-341, 2008.

HERWEIJER, C. et al. How do our coastal cities fare under rising flood risk? Catastrophe Risk Management, Southampton, p. 12-13, 2008.

INEG - INSTITUTO NAGIONAL DE ESTADÍSTICAS Y CENSOS. Población y demografia. In: INSTITUTO NAGIONAL DE ESTADÍSTICAS Y CENSOS. Quito: Inec, 2015. Disponible en: https:// bit.ly/3dSf79d. Acceso: 1 jan. 2015.

IPGG - INTERGOVERNMENTAL PANEL ON GLIMATE CHANGE. Summary for policymakers: a report of Working Group I of the Intergovernmental Panel on Climate Change. Cambridge: Cambridge University Press, 2001.

KRABILL, W. et al. Greenland ice sheet: increased coastal thinning. Geophysical Research Letters, Washington, DC, v. 31, n. 24, p. 1-4, 2004.

MCGRANAHAN, G. et al. The rising tide: assessing the risks of climate change and human settlements in low elevation coastal zones. Environment and Urbanization, Thousand Oaks, v. 19, n. 1, p. 17-37, 2007.

NICHOLLS, R.; MIMURA, N. Regional issues raised by sea-level rise and their policy implications. Climate Research, Oldendorf, v. 11, 5-18, 1998.

NICHOLLS, R.; TOL, R. S. J. Impacts and responses to sea-level rise: a global analysis of the SRES scenarios over the twenty-first century. Philosophical Transactions of the Royal Society A, London, v. 364, n. 1841, p. 1073-1095, 2006. 
OVERPECK, J. T. et al. Paleoclimatic evidence for future ice-sheet instability and rapid sea-level rise. Science, Lincoln, v. 311, p. 1747-1750, 2006.

PATZ, J. et al. Impact of regional climate change on human health. Nature, Berlin, v. 438, n. 7066, p. 310317, 2005.
RIGNOT, E.; KANAGARATNAM, P. Changes in the velocity structure of the Greenland ice sheet. Science, Washington, DC, v. 311, n. 5763, p. 986-990, 2006.

VELICOGNA, I.; WAHR, J. Measurements of timevariable gravity show mass loss in Antarctica. Science, Washington, DC, v. 311, n. 5768, p. 1754-1756, 2006. 